\title{
Evaluating Factor Structure of Persian Version of Lifelong Learning Assessment Tool using Ordinal Versus Quantitative Methods in Confirmatory Factor Analysis
}

\author{
Mahbube Abdollahi ${ }^{1}$, Zeynab Avazzadeh ${ }^{2}$, Maryam Salari $^{1}$, \\ Farid Zaeri ${ }^{3}$, Fateme Pourhaji ${ }^{4}$, Elham Shaarbaf Eidgahi ${ }^{5}$, \\ Zahra Khosravi Anbaran $^{6}$ and Fatemeh Zahra Karimi ${ }^{6}$ \\ ${ }^{1}$ Biostatistics Department, Tarbiat Modares University, Tehran, Iran. \\ ${ }^{2}$ Department of Epidemiology and Biostatistics, School of Public Health, \\ Zahedan University of Medical Sciences, Zahedan, Iran. \\ ${ }^{3}$ Biostatistics Department, Shahid Beheshti Univesity, Tehran, Iran. \\ ${ }^{4}$ Health Promotion/Health Education Department, Tarbiat Modares university, Tehran, Iran. \\ ${ }^{5}$ Department of Biostatistics and Epidemiology, Mashhad \\ University of Medical Sciences, Mashhad, Iran. \\ ${ }^{6}$ School of Nursing and Midwifery, Mashhad University of medical science, Mashhad, Iran.
}

DOI: http://dx.doi.org/10.13005/bbra/1937

(Received: 01 July 2015; accepted: 03 October 2015)

\begin{abstract}
In factor analysis, despite ordinal nature of the data, the assumption is that the data are normal quantitatively. The present study aimed to survey the factor structure of Persian Version of lifelong learning assessment tool JeffSPLL-MS using the most accurate method in confirmatory factor analysis. In this cross-sectional study, 430 students of Birjand University of Medical Sciences were selected randomly. Persian version of JeffSPLLMS tool was used. First, assuming data scale being quantitative, three assessment methods were used, and by assuming data are ordinal, three assessment methods were used in confirmatory factor analysis, and eventually, the above mentioned six methods were compared. For comparing different methods, CFI, TLI and RMSEA indices were used. The three-factor structure of Persian version of JeffSPLL-MS had acceptable fit to the data. In all of the six methods, model indices confirmed the model fit, and ordinal models with correction had better fit compared to quantitative models. The result of the current study confirmed three-factor structure of Persian version of lifelong learning assessment tool. Although in confirmatory factor analysis, the common approach, taking into account the scale of quantitative data and using the maximum likelihood method, however, the results of this study showed that taking an ordinal scale data will result in improvement of fit model parameters.
\end{abstract}

Keywords: Confirmatory factor analysis, ordinal scale, factor structure, JeffSPLL-MS.

In medical science, commitment to learning throughout the life is considered an important element. Nowadays, lifelong learning is critical for safe medical care and development in medical researches and biotechnological development and

\footnotetext{
* To whom all correspondence should be addressed. E-mail: zeynab.evazzadeh@gmail.com
}

especially in clinical medicine because of rapid expansion of information and medical technologies $^{1,2}$.

In addition, the importance of students' education as lifelong learners has been the center of attention for many specialized organizations such as association of medical colleges in the United States. Moreover, development of lifelong learning is advised in all recommendations given 
for the purpose of medical education improvement ${ }^{3}$.

Despite the emphasis on lifelong learning, there is no generally accepted definition. One of the definitions of lifelong learning proposed by community of initiative lifelong learning of Europe is: "lifelong learning is the potential development of human through an ongoing supportive process which stimulates people in acquiring knowledge, values, skills and learnings they need in their lifetime and they use them with self-confidence, creativity, and pleasure at all roles, conditions, and environments". Another definition of lifelong learning is a concept that includes a set of selfstarting (from behavioral viewpoint) and information search skills (ability) that induces a stable motivation in individuals for learning and the ability to recognize their own educational needs (metacognition) $)^{1,4}$.

From lifelong learning point of view, students have to be equipped with lifelong learning skills, information literacy, learning how to learn and how to search through information resources, and universities are required to provide the infrastructures for strengthening the process of learning, and help students institutionalize learning methods and acquire information literacy ${ }^{5}$.

Hojjat et al. designed Jeffspll- MS Scale (16) for measuring the lifelong learning among medical students in Jefferson Medical College in the United States. In this study, we intend to verify Persian version of the above scale by the most accurate method in confirmatory factor analysis ${ }^{6}$.

In most psychometric questionnairesbased studies, the questions are designed through the Likert scale. Despite of ordinal nature of data with Likert scale in ordinary factor analysis (confirmatory or exploratory), it is assumed that the data are quantitative with normal distribution. This study aimed to compare the quantitative and qualitative methods in confirmatory factor analysis (CFA) and then, to examine the factor structure of Persian version of lifelong learning assessment tool by a more accurate method.

The most common method in factor analysis is Maximum Likelihood (ML) method. In this method, it is assumed that the data (indexes) come from a multivariate normal distribution.

Non-normal data violate the multivariate normal distribution assumption for different reasons such as kurtosis, skewness, and censored and effective data and outliers. Simulation studies showed that the violation of the normality assumption leads to the standard error bias of parameters estimation, but has no effect on estimation of model parameters ${ }^{7}$.

In case of violation of the normality assumption of data, standard errors can be moderated using specific statistical methods. For non-normal quantitative data, some methods such as Maximum likelihood mean adjusted (MLM) and mean and variance-adjusted maximum likelihood (MLMV) are introduced. In these two methods, factor loadings and standard errors are equal. The only difference is the method of adjusting chisquare statistic. If indexes are nominal or ordinal or a combination of nominal, ordinal and continuous, some methods such as the Weighted Least Square (WLS), Weighted least square mean adjusted (WLSM), and mean and Weighted least square mean and variance adjusted (WLSMV) can be used. Both WLSM and WLSMV estimators are robust and generate identical estimation and SE. The only difference lies in chi-square statistic adjusting method ${ }^{8}$.

\section{MATERIALSANDMETHODS}

This research is a cross-sectional study. The statistical population of the study consisted of all students at the Birjand University of Medical Sciences in 2013. The sample consisted of 430 students selected through proportional stratified random sampling in which the colleges were considered as stratifications, and the proportion of each subgroup is determined based on the whole population. First, the purpose of the study was explained for individuals and if they agreed to participate in the study, the questionnaire was completed by them. At first, in order to localize the questionnaire, a copy of the scale was sent to Iran by the designer of the questionnaire, and then researchers translated it to Farsi and matched it with the original text and had it confirmed by the designer.

The scale included 14 items of four options for each item so that every item is scored between 1 up to 4 . Subjects read every item and answered them in a range of strongly agree ${ }^{1}$ to strongly disagree ${ }^{4}$. The highest score a subject 
can achieve in this scale is reported 56 and the lowest score is reported $14{ }^{6}$.

Results of the factor analysis showed that this scale consisted of three factors including beliefs associated with learning and motivation (items 1, 2, 3, 7, 8, 9, and 11), information search skills (items 5, 6, 10, and 14) attention to learning opportunities $(4,12,13)$.

In order to study the factor structure of Persian version of lifelong learning assessment tool by a more accurate method in confirmatory factor analysis, maximum likelihood estimation (ML), maximum likelihood mean-adjusted (MLM), and maximum likelihood mean and variance-adjusted (MLMV) methods were taken into account in quantitative form; and Weighted Least Square(WLS), Weighted least square meanadjusted (WLSM), Weighted Least Square Mean and Variance (WLSMV) estimation methods were used in ordinal form. Eventually, the results of the six methods were compared. To compare the quantitative and qualitative methods, Comparative fit index (CFI), Tucker-Lewis index (TLI), and Root mean square error of approximation (RMSEA) ${ }^{7}$ were used, Mplus software version 6 was used for the analyses $^{9-10}$.

CFI varies from zero to one .(If the stat values are out of this range, less than zero values are considered zero and more than 1 is considered 1). Zero CFI shows the weakest fitness and the value of one shows the best fitness. Based on an experimental rule, the value of 0.9 or more is appropriate for this index. CFI index is a good one for small sample sizes, too ${ }^{8,11}$.

Similar to CFI, a zero value of TLI shows the poor fitness, while TLI value of 1 shows the best fitness. If the stat values are out of this range, less than zero values are considered zero and more than 1 is considered 1. Based on an experimental rule, the value of 0.9 or more is appropriate for this index $^{8,12}$.

Table 1. Comparison of the indices resulted from quantitative and qualitative models to the data

\begin{tabular}{lcccc}
\hline Model & Model parameters estimation method & RMSEA & CFI & TLI \\
\hline \multirow{2}{*}{ Quantitative model } & ML & 0.08 & 0.86 & 0.83 \\
& MLM & 0.07 & 0.87 & 0.84 \\
\multirow{3}{*}{ Ordinal Model } & MLMV & 0.06 & 0.87 & 0.84 \\
& WLS & 0.11 & 0.81 & 0.76 \\
& WLSM & 0.09 & 0.94 & 0.93 \\
& WLSMV & 0.08 & 0.92 & 0.9 \\
\hline
\end{tabular}

RMSEA index of $\leq 0.05$ indicates a good fit; $0.05 \leq$ RMSEA $\leq 0.08$ represents a reasonable and acceptable fit; $0.08 \leq$ RMSEA $\leq 0.1$ is average fitness; and $0.1<$ RMSEA indicates a poor fitness of the model ${ }^{13}$.

It is noteworthy that Kaiser-Meyer-Olkin (KMO) statistic needs to be calculated prior to confirmatory factor analysis in order to ensure the adequacy of sampling. If KMO statistic is greater than 0.8 , it indicates adequate sampling. In addition, Bartlett's sphericity test is performed to ensure the justifiability of factoring. In case of rejection of the null hypothesis in Bartlett's test, factoring is justifiable ${ }^{17-18}$.

\section{Findings}

This study was conducted on 430 students of Birjand university of Medical Science.
However, with the loss of 32 questionnaires, 398 questionnaires were statistically analyzed. The mean age of students was $36.2 \pm 12.22$. The mean scores of lifelong learning and three subscales including learning-related beliefs and motivation, information search skills, and attention to learning opportunity in students were reported $40.72 \pm 6.06$, $18.61 \pm 3.05,13.80 \pm 2.53$, and $8.37 \pm 1.75$, respectively. In accordance with analyzing JEFFSPLL-MS factor structure, KMO statistic is calculated in order to ensure adequate sampling. In addition, to examine data correlation matrix not being zero in population or justifiable factoring, Bartlett sphericity test was conducted. Since KMO statistic is greater than $0.8(0.852)$ and the null hypothesis is rejected with stat value of 1418 at $5 \%$ level, sampling enjoys adequacy and factor extraction can be used. 


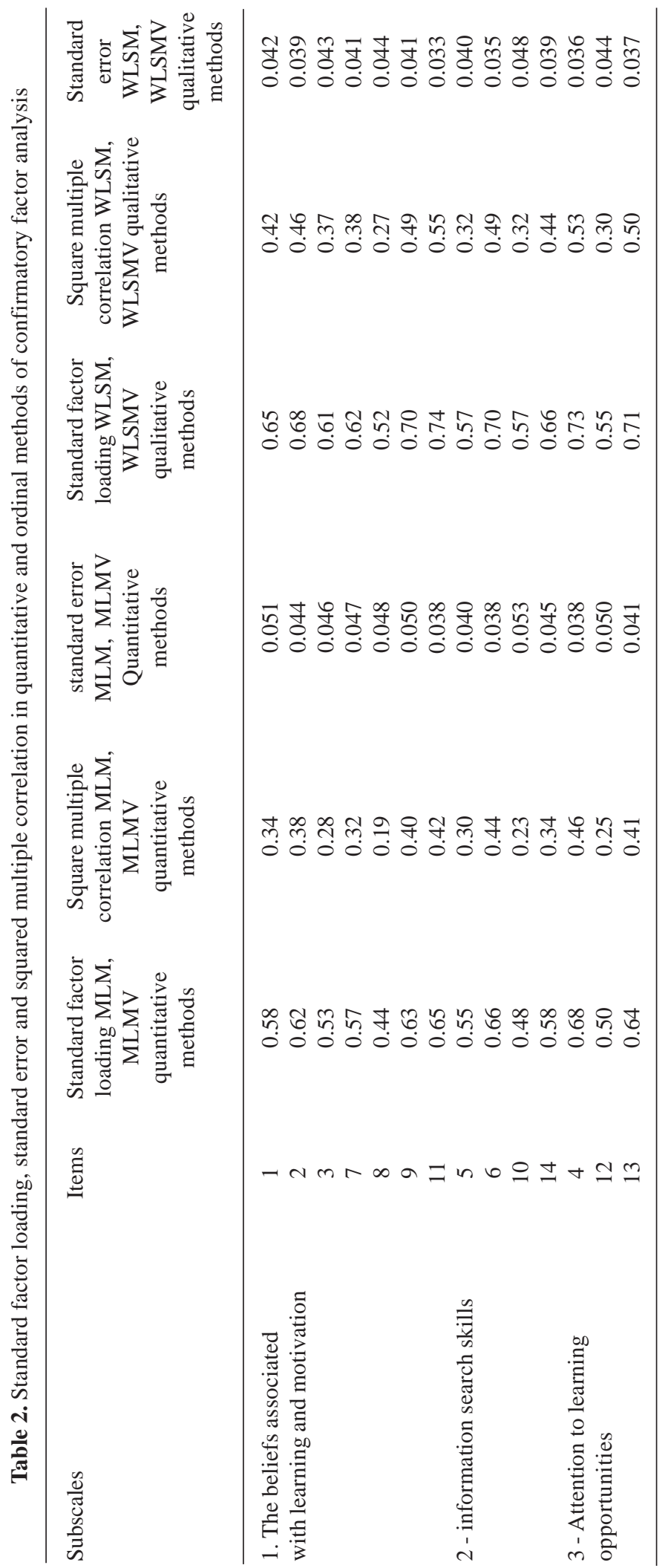


The results of ML, MLM, MLMV and WLS, WLSM, WLSMV fitting methods are shown in Table 1. For goodness of fit of the model, RMSEA needs to be between zero and 0.1. Values less than 0.05 represent a good model fit for this index. As shown in Table 1, RMSEA is less than 0.1 in both quantitative and qualitative methods (except for WLS qualitative method). Therefore, model fitness is relatively perfect. Values higher than 0.9 represent a perfect fit of the model for CFI and for TLI indices. As shown in Table 1, these indices are equal to 0.9 in WLSMV ordinal method. In general, TLI and CFI indices are higher in ordinal methods (WLSM, WLSMV) than quantitative methods (ML, MLM, MLMV). The results of Table 1 show that WLSMV ordinal method enjoys more appropriate fitness than the others. Therefore, the JEFFSPLLMS three -factor structure is approved in the sample of Iranian students. For a closer look, we compare the standard errors and factor loading in MLM, MLMV quantitative methods, as well as WLSMV, WLSM qualitative methods.

The results of MLM and MLMV quantitative methods and WLSM and WLSMV ordinal methods are shown in Table 2. All items are significant on their factors (p. value $<0.05$ ). Comparing the standard error of factor loadings indicates that $\mathrm{SE}$ is smaller in ordinal methods than quantitative methods, i.e., ordinal methods are more accurate than quantitative methods.

Larger factor loading indicates which item represents is better related to its subscale. The results of ordinal methods suggest that, in the first subscale, (beliefs related to learning and motivation), items 9 and 11; in the second subscale (information search skills), items 6 and 14; and in the third subscale (attention to learning opportunities), items 4 and 13 are better indices for these subscales. The results can be seen in quantitative methods as well. However, standard factor loadings are greater in ordinal methods than quantitative methods.

The square of multiple correlation coefficients of quantitative and ordinal methods results show that the first subscale represents 34\% of the variance of the first items in the quantitative method, and the first subscale represents $42 \%$ of the variance of the first item in ordinal method. Similarly, for the other subscales and items, the results showed that using ordinal method explains a greater percentage of items' variances by subscales.

\section{DISCUSSION}

The purpose of this study was comparing quantitative data scales versus ordinal data scales in Likert type questionnaires. Furthermore, the assessment of the Farsi version of the lifelong learning tools can be performed in a more accurate way.

In practice, CFA with continuous variables (and in general, ordinal variables with 5 categories or more) does not face serious problems if the assumption of normality of variables is violated $^{14}$.

However, in case of non-normal variables, the standard errors become very small (therefore, the tests of significant path coefficients lead to an increase in type 1 error). It seems that adjusted standard error introduced by Satorra and Bentler (1994) is a good general method for analyzing nonnormal data ${ }^{15}$.

Hutchinson and Almas (1998) showed that when variables are in ordinal scale with large number of categories, such as 7 categories Likert scale, assuming continuous data creates no problem in statistical analyses. However, when the number of categories is small, for example 2 to 4 categories, it is better to analyze with qualitative method ${ }^{15}$.

The results of this study showed that taking ordinal scale into account in data leads to a more accurate fitness of the model to the data; furthermore, the validity of the Farsi version of lifelong learning assessment tool is established as well. In other words, subscales extracted from the data related to the Farsi version match the original version perfectly. Therefore, this questionnaire is a useful tool to evaluate lifelong learning tool.

\section{CONCLUSION}

In this study, the results showed that although the model parameters are within the limits that confirm model fitness in all six confirmatory factor analysis. Also, considering ordinal scale for the data would lead to improvement of model indices so that the TLI and CFI indices are about 0.1 higher in ordinal methods (WLSM, WLSMV) 
than quantitative methods (ML, MLM, MLMV). On the other hand, RMSEA indicator is less than 0.1 . ). In this way, using ordinal methods with correction, we achieved a more precise fitness for factor structure of Farsi version of lifelong learning tool. In addition, the results showed that Farsi version of lifelong learning tool is confirmed for Iranian students.

\section{ACKNOWLEDGMENTS}

Hereby we would like to express our gratitude to Professor Mohammad Reza Hojjat, Professor of Jefferson medical college for his valuable assistance and guidance, we also like to express our sincere thanks towards all the students participating in this project.

\section{REFERENCES}

1. Iranian National Commission for UNESCO. [Karegahe amouzeshiye meliye mafahim va nezamhaye jaded dar zamineye yadgiriye madamolomr]. Tehran Cluster Office; 2013. [Persian] [cited 2015 January 14] Available from: http://www.unesco.org/new/fileadmin/ MULTIMEDIA/FIELD/Tehran/pdf/ LLL1114Feb13Finalreport070813UTCONatcom-Farsi.pdf

2. Hojat M, Veloski JJ, Gonnella JS. Measurement and Correlates of Physicians’Lifelong Learning. Acad Med. 2009; 84(8): 1066-1074.

3. Hojat M, Gonnella JS, Veloski JJ. Predictors and Outcomes of Physician Lifelong Learning. [cited 2015 January 14] Available from: http:// www.researchgate.net/publication/ 33684996_Predictors_and_Outcomes_ of_Physician_Lifelong_Learning

4. Hojat M, Veloski JJ, Gonnella JS. Physician Lifelong Learning: Conceptualization, measurement and correlates in full - time clinicians and academic clinicians. Handbook of Lifelong Learning Developments. Nova Science Publishers. 2009.
5. Karimi S, Nasr Esfahani A, Boghratian K. [Manabe va khadamate elmi pajouheshie mowrede niaze daneshgah baraye tahaghoghe yadgiriye madamolomr]. Faslnameye ketab. 2005; 65: 163-184. [Persian]

6. Wetzel AP1, Mazmanian PE, Hojat M, Kreutzer KO, Carrico RJ, Carr C, Veloski J, Rafiq A.Measuring medical students' orientation toward lifelong learning: a psychometric evaluation. Acad Med. 2010; 85(10 Suppl):S414.

7. Sharma S. Applied multivariate techniques: John Wiley \& Sons, Inc.; 1995

8. Wang J, Wang X. Structural equation modeling: Applications using Mplus: Wiley; 2012.

9. Byrne BM. Structural equation modeling with LISREL, PRELIS, and SIMPLIS: Basic concepts, applications, and programming. Psychology Press LTd; 2006.

10. Raykov T, Marcoulides GA. A first course in structural equation modeling: Routledge; 2006.

11. Herberg PY, Beauducel A. On the Performance of Maximum Likelihood Versus Means and Variance Adjusted Weighted Least Squares Estimation in CFA, Structural Equation Modeling. A Multidisciplinary Journal 2009; 13(2): 186-203.

12. Hooman HA. Structural equation modeling with LISREL application. Samt publication; 2010.

13. Loehlin JC. Latent variable models: An introduction to factor, path, and structural equation analysis: Psychology Press; 2004; ch:2, p:69.

14. Hutchinson SR, Olmos A. Behavior of descriptive fit indexes in confirmatory factor analysis using ordered categorical data. Structural Equation Modeling: A Multidisciplinary Journal 1998; 5(4):344-64.

15. Curran PJ, West SG, Finch JF. The robustness of test statistics to nonnormality and specification error in confirmatory factor analysis. Psychological methods 1996;1(1):16.

16. Soltani Arabshahi S, Naeimi L. [Assessment of the current situation of - self-directed learning skills in medical students]. Razi Journal of Medical Sciences. 2013; 20(113): 10-19. [Persian]. 